\title{
Self-select target neighboring base station assisted handover for natural disaster in LTE-A network
}

\author{
Azita Laily Yusof, Ainnur Eiza Azhar, Norsuzila Ya'acob \\ Wireless Communication Technology Group, Faculty of Electrical Engineering, Universiti Teknologi MARA, Malaysia
}

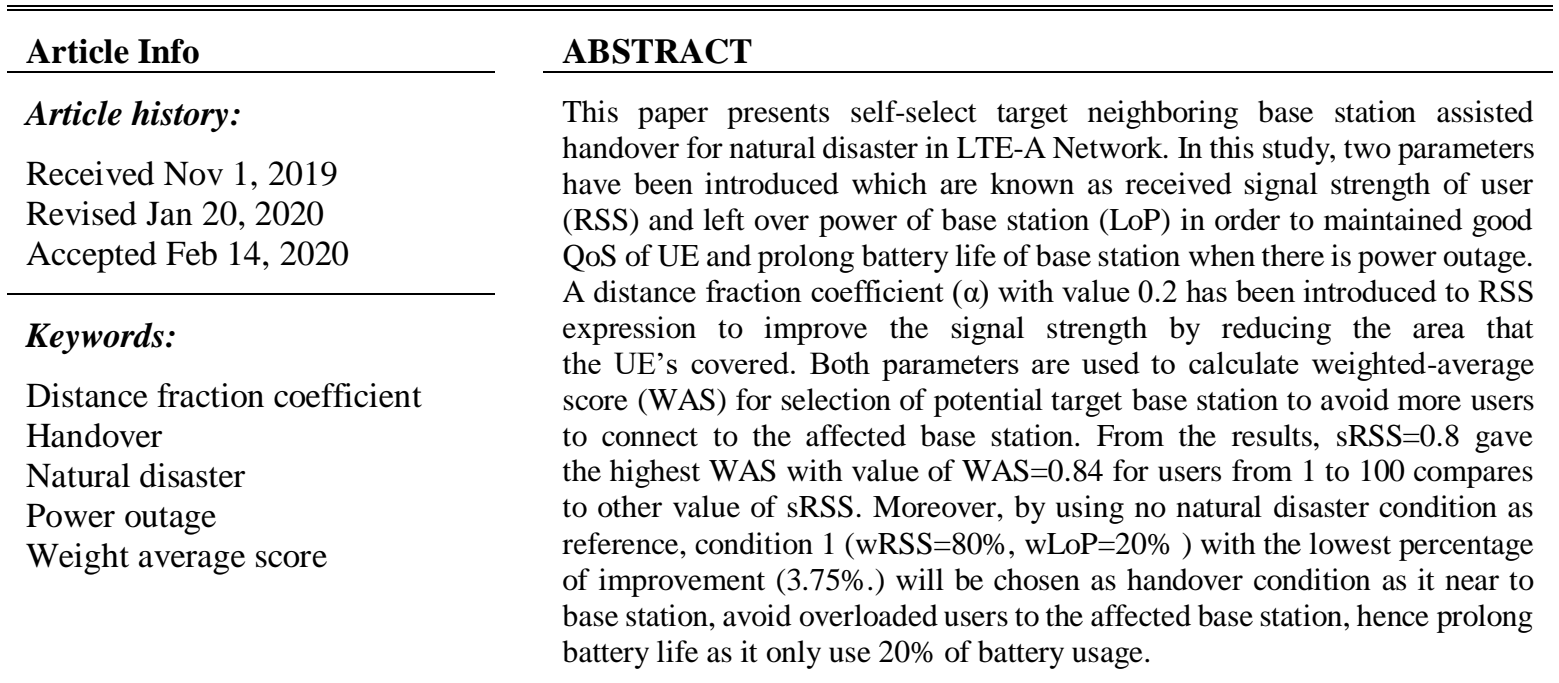

Copyright $(2020$ Institute of Advanced Engineering and Science. All rights reserved.

\section{Corresponding Author:}

Azita Laily Yusof,

Wireless Communication Technology Group,

Faculty of Electrical Engineering,

Universiti Teknologi MARA, Shah Alam, Malaysia.

Email: laily012001@yahoo.com

\section{INTRODUCTION}

Natural disasters such as flood, landslide, earthquake, tsunami and other cannot be predicted and the after-effect will bring major destruction and damage. Several issues have been highlighted by previous researchers which are rescue operation; awareness prediction and damage of mobile communication. For rescue operation, in [1], the author has proposed a navigation system using D2D communications and GPS for rescue operation. The D2D provides public protection and disaster relief (PPDR) and national security and public safety (NSPS) services which capable to support local services although some or all of the network nodes become damage or faulty due to natural disaster. The advantage of the approach is has allocation of frequencies. In [2, 3], the authors proposed unmanned and autonomous vehicle (UAV), that can support humans in the affected area hence significantly reduce their radius of rescue operation. The technique provides data gathering and processing, overviews of visual situation and sending high-level commands to commanders for coordinating rescue missions. In addition, it also can automatically take decisions on its own during unpredictable situations [3]. Also, in [4], the author has review optimal energy efficient path planning for unmanned air vehicles. However, this technique is not cost-effective as it uses unmanned vehicles and high range communication infrastructure. Other than that, author in [5] has developed an Event detection obtained from mobile phone users. It can detect both positive (i.e: festivals) and negative events (i.e: natural disasters and road accidents) by acquire location, areas and days of Events occurred from mobile phones carriers. 
The technique is using GPS and it can accurately detect the location however it makes the mobile phone's battery drain faster due to the GPS requires more power consumption. Meanwhile, authors in $[6,7]$ using geographical information system (GIS) as a technique to identify the disasters areas accurately by developing a tool that provides quick response and can be useful for post-earthquake management. It also can be function as an alternative to the GPS. However, this technique depends on left over power of mobile phones to operate as power outage may occur at the affected areas. In addition, paper in [8] presented a hybrid network architecture that integrates LTE and satellite technologies for PPDR that has easy connectivity, extended coverage and high performance guarantees. However, it also uses satellite that needs high power to operate. Also, in [9], the author has proposed a tool for monitoring and tracking end-user health conditions during disasters by using Microphone, speakerphone, on-device camera, accelerometer, gyroscope, compass, radio frequency (LTE), smartphone disaster recovery system (SDRS) Application. If no coverage, it will forward the information using D2D. However, the technique is only health monitoring application. Another paper [10] has proposed computational method for angle tracking radar. The results of theoretical and simulation-based are identical to each other. However, this paper focuses only on mathematical operation and not for rescue operation using angle tracking radar.

On issue of prediction awareness, author [11] proposed a Disaster Management using WSN Technology using flood and earthquake sensors for prediction awareness. It uses central node to receive information from the sensors and process it by compare the information with reference voltage. Then, the data goes to the nearest base station. However, this technique has disadvantage which it depend on central node and does not has backup. On the other hand, author in [12] has applied the social media to collect disaster data by using driven approach for disaster response through sentiment analysis. This technique collects disaster data from social networks and categorizes them according to the needs of the affected people using machine learning algorithm. Also, author [13] has proposed a combination of the GIS and Mobile IT-based Android as an early warning system for large-scale incidents by using social media. The benefits by using the technique are it provides help to the victims by knowing the exact location for help and manage the needs of the people in disaster affected. However, because it only depends on information from social media; it did not have event's priority levels i.e. low, medium and high risks to avoid unnecessary help and waste of resources. Subsequently, researchers in [14] has proposed an integrated early warning system (iEWS) known as mKRISHI® Fisheries for prediction awareness, minimizing the vulnerability and subsequent losses for ocean and flood disasters. The technique used Wind and Wave information generated by INCOIS and it can identify the risk zones, occurrence date and time and help the fishers by reducing the risk exposure and saving lives. However, the information received will be channeled to mobile phones.

For damage of mobile communication issue, it can be disrupted due to critical hardware failure and power outage. Although base station has battery backup to operate, the battery life is limited [15]. If there is no countermeasure from the network operator for this issue, remaining power of base station to operate becomes critical, hence, traffic congestion will occur. Previous researchers have studied several techniques to overcome traffic congestion in limited power due to natural disaster. Author in [16] proposed utilizing Raspberry Pi Single Board Computing devices running Docker to allow for the rapid deployment of communications system in areas where little or no communications infrastructure exists especially for natural disaster. The benefit of this technique is it used software defined networks (SDN) as a command and control system. In addition, it also has the ability to remotely enable network elements and provide scalability and programmability. However, both techniques offered a wide area for environmental monitoring and data collection tasks which means signal strength of the users might decreased due to far from base station. Mean while, author in [17] applied SON techniques include self-configuration of power, physical cell identifiers (PCI), and automatic neighbor relation (ANR), as well as self-optimization of mobility robustness, and coverage and capacity. It has advantage due to small cell only require low power to operate. However, the technique does not control handover traffic and only focuses on self-configuring during power outage. Other than that, several researchers have proposed contingency cellular network $(\mathrm{CCN})$ for emergency communication by connecting disconnected base stations together with wireless links and constructing a multi-hop cellular network. Author in [18] has proposed Bandwidth Allocation of $\mathrm{CCN}$ for quick response and efficient during disasters, however it has high complexity due to heuristic algorithm. In addition, authors in $[15,19]$ also have proposed $\mathrm{CCN}$ technique, however, the damage of a disaster may not evenly distribute and time consuming.

Other than that, several papers investigate on energy saving during natural disasters. In [20], the author has proposed a scheme that can help to limit the number of new clients connecting to available eNBs, consequently reducing per-eNB load and allowing eNBs to remain alive for longer. The technique use Wi-Fi tethering as it consumes much low power however it operates on data network. In addition, the author [21] has employed energy harvesting $(\mathrm{EH})$ at the relay with simultaneous wireless information and power transfer to prolong the lifetime of energy constrained network using D2D communication. Also, in [22], the author using 
D2D Communication for Disaster Recovery that adopts multi-hop D2D relays for routing protocol. It is proved that this technique has high efficiency in order to extend cellular coverage in the case of a network infrastructure failure or a natural disaster. The advantage of this method is robust, energy saving and time saving for communication; however, it has higher complexity. Previous papers in [23, 24] have proposed an LTE-A UE-controlled and eNB- assisted reliable handover scheme for natural disaster situations. It discourages the arrival of new traffic to an already overloaded eNB after post-disaster. By using the technique, the UE can self-select the best NeNB to handover with based on the weighted averaging of scores (WAS) for each NeNB assigned against the two different parameters, namely, the leftover power (LoP) of NeNBs in the disaster affected area and the UE's distance of motion (DoM) relative to a NeNB. The advantage of the technique is the UE can self-assign individual scores to the different NeNBs and selects the TeNB for the handover activity. In addition, the signal strength (RSS) of a UE was used as handover threshold bound to limit the arrival of new traffic to an already overloaded eNB. However, this technique has disadvantage which is it used complicated algorithm using UE's movement paths known as Angle of Divergence (AoD) by characterized the angle value from $0^{\circ}$ to $180^{\circ}$. Where: $\mathrm{AoD}=0^{\circ}$ is for MS which moving exactly towards the potential target base station (PTBS) while $\mathrm{AoD}=180^{\circ}$ is for the MS which moving exactly away from the PTBS. Other than that, in [25], the author has employed SDN-based approach for providing an energy-efficient heterogeneous communication network for victims in disaster scenarios. The results proved the flexibility and provided small delay of the proposed system. However, the technique is using Bluetooth and Wi-Fi which have different physical interface make it difficult to interact due to different protocols.

This paper investigates the continuity of the paper $[23,24]$ which need to identify parameters that can limit the arrival of new traffic to an already overloaded eNB by diverting their handover to lightly loaded nearby eNBs. In this work, the RSS was chosen as a parameter as it determines the values of the transmission power, the distance between the transmitter and the receiver, and the radio environment. In addition, it also highly correlated to the transmitter's location [26]. Together with LoP, the WAS of natural disaster can be calculate for handover to the target eNBs. Hence, it can maintain good QoS with the users located near to base station and prolong the battery life by reducing the power consumption. The rest of the paper is organized into five different sections. Section 2 presents the brief explanation of parameter RSS and LoP and their mathematical expression and formulation of proposed UE-controlled Intelligent Handover Strategy for natural disaster scenarios. Section 3 deals with results and discussion and finally conclusion is drawn in section 4.

\section{METHODOLOGY}

This section explained the brief explanation of mathematical expression of RSS, LoP and WAS. Previously, we investigated new parameter namely as distance fraction coefficient $(\alpha)$ on received signal strength (RSS) of a user for natural disasters scenario [27]. By applying the parameter on RSS, it increases the value of RSS, so the UE with good network performance can handover to neighboring base stations than the affected base station. The modified received signal strength $\left(R S S_{\text {modified }}\right)$ in decibel can be expressed as:

$$
R S S_{\text {modified }} P_{t}+G_{t}+G_{r}-P L_{\text {modified }}
$$

where: $P_{t}$ is transmitted power of a BS, $G_{t}$ is transmitter antenna gain, $G_{r}$ is receiver antenna gain and $P L_{\text {modified }}$ is path loss between UE and BS. The modified path loss for this work is adopted two-ray path loss and can be defined as:

$$
P L_{\text {modified }}=20 \times \log \left(\alpha \times d^{2} / h_{t} \times h_{r}\right)
$$

where: $\alpha$ is distance fraction coefficient, $d$ is distance from UE to BS, $h_{t}$ is transmitter's antenna height and $h_{r}$ is receiver's antenna heights. In [27], the value of $\alpha$ was varied from 0.2 to 0.8 . From the results, $\alpha=0.2$ was chosen as the optimal value because it gave the highest RSS value of UE compare to others. It is because the $\alpha$ affects the area covered, which reduced the user's area serving hence giving it much lower path loss value to the system.

As stated in [23], left over power (LoP) of a battery can be expressed as:

$$
L o P=[(N-n / N)] \times \text { Rated power of the battery }
$$

where: $\mathrm{n}$ is number of connection and $\mathrm{N}$ is maximum of $\mathrm{N}$ connections at any time without any degradation in the QoS. When the number of connections is less than maximum connections of base station, the maximum 
power drawn from the battery is less. The base station can support some more connections without degradation of QoS. When $n=N$, the LoP is equal to 0 . The LoP has maximum power available when $n=0$. The UE will receives different information of LoP for different neighboring base station, thus, it assigns them to individual scores namely as SLoP. The least LoP is the lowest score. For sLoP, the equation can be expressed as:

$$
S_{L o P}=(N-n) / N
$$

where: $\mathrm{n}=$ number of connection and $\mathrm{N}=$ maximum of $\mathrm{N}$ connections at any time without any degradation in the QoS. The sLoP was fixed in range $0 \leq \mathrm{SLoP} \leq 1$. When more subscribers attached to an eNB, the fewer users are inviting to pass through it. This technique will make the affected base station not overloaded; hence help to prolong the battery life.

For handover activity, the UE calculated both parameters Lop and DoM. The highest WAS which the NeNB receive will be select as the target eNB (TeNB). The SWAS (NeNBi) for each NeNB can be expressed as:

$$
S_{W A S}\left(N e N B_{i}\right)=S_{L o P}\left(N e N B_{i}\right) \times W_{L o P}+S_{D o M}\left(N e N B_{i}\right) \times W_{D o M}
$$

where: $S_{L o P}\left(N e N B_{i}\right)$ is LoP score for neighboring eNB, $S_{D o M}\left(N e N B_{i}\right)$ is DoM score for neighboring eNB, $W_{L O P}$ is weight of LoP, and $W_{D o M}$ is weight of DoM. The weight is fixed to $0 \leq \mathrm{WLoP}, \mathrm{WDoM} \leq 1$ with condition:

$$
W_{L o P}+W_{D o M}=1
$$

For this work, the UE will calculate SWAS for each NeNB, then, it will select the highest score for handover. By applying the RSS instead the DoM, it reduced the serving area, hence improves the user signal strength. The modified SWAS for each NeNB is taken from (5) and can be expressed as:

$$
S_{W A S}(\text { modified })=S_{L o P}\left(N e N B_{i}\right) \times W_{L o P}+S_{R S S_{-} \text {modified }}\left(N e N B_{i}\right) \times W_{R S S}
$$

where: $S_{L o P}\left(N e N B_{i}\right)$ is LoP score for neighboring eNB as (4), $S_{R S S_{-} \text {modified }}\left(N e N B_{i}\right)$ is modified RSS score for neighboring eNB as Table $1, W_{L o P}$ is weight of LoP and $W_{R S S}$ is weight of RSS as shown in Table 2.

Table 1 explain about the score for RSS which value $S_{R S S \_ \text {modified }}=1.0$ gives the characterization of the user is moving exactly towards the potential target base station (PTBS). Meanwhile, $S_{R S S_{\text {_modified }}}=0$ gives the description of the user is moving exactly away from the PTBS. Table 2 describes the score of weights for RSS and LoP. The total of both weights is equal to 1 . For Condition 1, it shows that no natural disasters happen.

\begin{tabular}{|c|c|c|}
\hline$S_{R S S \_ \text {modified }}$ & Characterization of the motion of MS & Distance $(\mathrm{m})$ \\
\hline 1.0 & MS is moving exactly towards the PTBS & 0 \\
\hline 0.8 & $\begin{array}{l}\text { The MS is moving towards the PTBS but its progressive movement towards the PTBS is } \\
\text { far than the highest possible value at RSS }=1.0\end{array}$ & $1<\mathrm{d}<50$ \\
\hline 0.6 & $\begin{array}{l}\text { The MS is moving towards the PTBS but its progressive movement towards the PTBS is } \\
\text { far than the possible value at RSS }=0.8\end{array}$ & $51<\mathrm{d}<100$ \\
\hline 0.4 & $\begin{array}{l}\text { The MS is moving towards the PTBS but its progressive movement towards the PTBS is } \\
\text { far than the possible value at RSS }=0.6\end{array}$ & $101<\mathrm{d}<150$ \\
\hline 0.2 & $\begin{array}{l}\text { The MS is moving away from the PTBS but its regressive movement away from the } \\
\text { PTBS is far than the highest possible value, which occurs at RSS }=0 \text {. }\end{array}$ & $151<\mathrm{d}<200$ \\
\hline 0 & The MS is moving exactly away from the PTBS & $>200$ \\
\hline
\end{tabular}
Meanwhile the weight for RSS is inversely proportional to the weight of LoP. Meanwhile, Table 3 shows the value for simulation part. Table 3 shows the simulation parameter used for (7).

Table 1. The propose RSS score, characterization of MS's motion and distance 
Table 2. The propose weight of RSS and LoP and its description

\begin{tabular}{|c|c|c|}
\hline Condition & wRSS: wLOP & Description \\
\hline 1 & 100\% wRSS: $0 \%$ wLOP & $\begin{array}{l}\text { Fully dependable on signal strength of UE based on distance near to target base } \\
\text { station. In addition, no natural disaster incidents occur. }\end{array}$ \\
\hline 2 & $80 \%$ wRSS: $20 \%$ wLOP & $\begin{array}{l}\text { Location of UE is near to BS with only small percentage of battery backup in natural } \\
\text { disaster incidents. }\end{array}$ \\
\hline 3 & $60 \%$ wRSS: $40 \%$ wLOP & $\begin{array}{l}\text { Location of UE is far from BS than condition } 2 \text { with slightly better battery backup } \\
\text { in natural disaster incidents. }\end{array}$ \\
\hline 4 & $40 \%$ wRSS: $60 \%$ wLOP & $\begin{array}{l}\text { Location of UE is far from BS than condition } 3 \text { with good battery backup in natural } \\
\text { disaster incidents. }\end{array}$ \\
\hline 5 & $20 \%$ wRSS: $80 \%$ wLOP & $\begin{array}{l}\text { Location of UE is far from BS than condition } 4 \text { with better battery backup in natural } \\
\text { disaster incidents. }\end{array}$ \\
\hline 6 & $0 \%$ wRSS: $100 \%$ wLOP & $\begin{array}{l}\text { Fully dependable on battery backup in natural disaster incidents. In addition, no } \\
\text { signal strength of UE is taken into account. }\end{array}$ \\
\hline
\end{tabular}

Table 3. Simulation parameter

\begin{tabular}{cc}
\hline Parameter & Value \\
\hline Pt_dbm in natural disasters & $46 \mathrm{dBm}[28]$ \\
Gt_dbi & $15(\mathrm{dbi})$ for downlink \\
Gr_dbi & $0(\mathrm{dbi})$ for downlink \\
$\mathrm{ht}$ & $30 \mathrm{~m}$ \\
$\mathrm{hr}$ & $1.5 \mathrm{~m}$ \\
$\mathrm{~d}$ & minimum distance $35 \mathrm{~m}$ \\
$\alpha$ & $0<\alpha<1$ \\
\hline
\end{tabular}

\section{RESULTS AND ANALYSIS}

This section explained the simulation results and its analysis for propose $S_{W A S}$ (modified). Figure 1 shows the WAS Neighboring eNB versus Number of subscribers for weight of RSS (wRSS) is $80 \%$ and weight of LOP (wLOP) is 20\%. By applying number of subscribers from 1 to 100, different value of sRSS which are $0.2,0.4,0.6$ and 0.8 were investigated on WAS neighboring value. From the result, $\mathrm{sRSS}=0.8$ gives the highest value of WAS neighboring eNB. It is because of the subscribers were located near to the target base station. This value will be using as handover threshold for handover algorithm in natural disaster incidents. Other than that, the value of WAS Neighboring eNB is decreases when the value of sRSS is reduces. It is because of the location of the subscribers become far from the target base station. In term of number of subscribers, it is shows that number of subscribers affect the value of WAS where the value of WAS decreases when the number of subscribers increases. It is because of less traffic loads consume less battery of eNB thus give the high value of WAS compares to high traffic loads. In addition, a heavy load at an eNB also means fewer the number of new connections it invites to pass through it. Table 4 shows the value of WAS for 1 user using $\operatorname{sRS}=\{0.8,0.6,0.4$ and 0.2$\}$. The sRSS with the highest WAS will be choose for the handover condition in natural disaster.

Figure 2 shows the WAS neighboring eNB versus number of subscribers with sRSS is fixed to 0.8 and different types of scenarios. For 100\% wRSS, $0 \%$ wLOP, the value of WAS is constant to any number of subscribers due to location of subscribers are near to the target base station. For $0 \%$ wRSS, 100\% wLOP, it represents the maximum value of LoP to select TeNB and to sustain UE's own communication reliability as distance of UE from the target base station is not taken into account. For other conditions, the value of WAS for $80 \%$ wRSS, $20 \%$ wLOP is lowest compare to others because of distance of subscribers are near to the target base station, hence QoS at UEs is improved. However, the value of wLoP is the lowest for other conditions because only small percentage of battery backup is used. The weights of wLoP need to be higher, as more subscribers can be attached to the target base station. However, signal strength of UE must be high to improve QoS of the UE. Table 5 shows the value of WAS for 1 user by conditions in Table 2. The WAS value with the lower percentage of improvement $(3.75 \%)$ compares to no natural disaster was chosen for the best conditions for the handover condition as it near to reference condition which is no natural disaster scenario.

Table 4. Results of value of WAS for 1 user using sRSS $=\{0.8,0.6,0.4$ and 0.2$\}$

\begin{tabular}{cc}
\hline sRSS & WAS Value \\
\hline 0.8 & 0.84 \\
0.6 & 0.68 \\
0.4 & 0.52 \\
0.2 & 0.36 \\
\hline
\end{tabular}


Table 5. Results of value of WAS for 1 user by conditions in Table 2

\begin{tabular}{ccc}
\hline Conditions & WAS Value & $\% \Delta$ \\
\hline 100\% wRSS: $0 \%$ wLOP & 0.80 & - \\
80\% wRSS: $20 \%$ wLOP & 0.83 & $3.75 \%$ \\
60\% wRSS: $40 \%$ wLOP & 0.88 & $10 \%$ \\
40\% wRSS: $60 \%$ wLOP & 0.91 & $13.75 \%$ \\
20\% wRSS: $80 \%$ wLOP & 0.96 & $20 \%$ \\
0\% wRSS: $100 \%$ wLOP & 1.0 & $25 \%$ \\
\hline
\end{tabular}

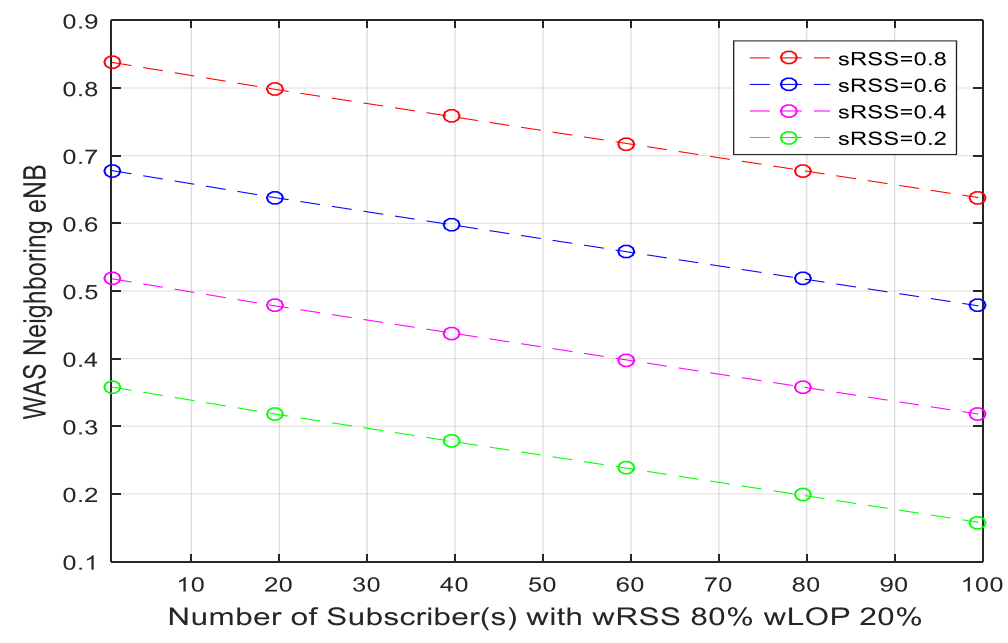

Figure 1. WAS neighboring eNB vs number of subscribers

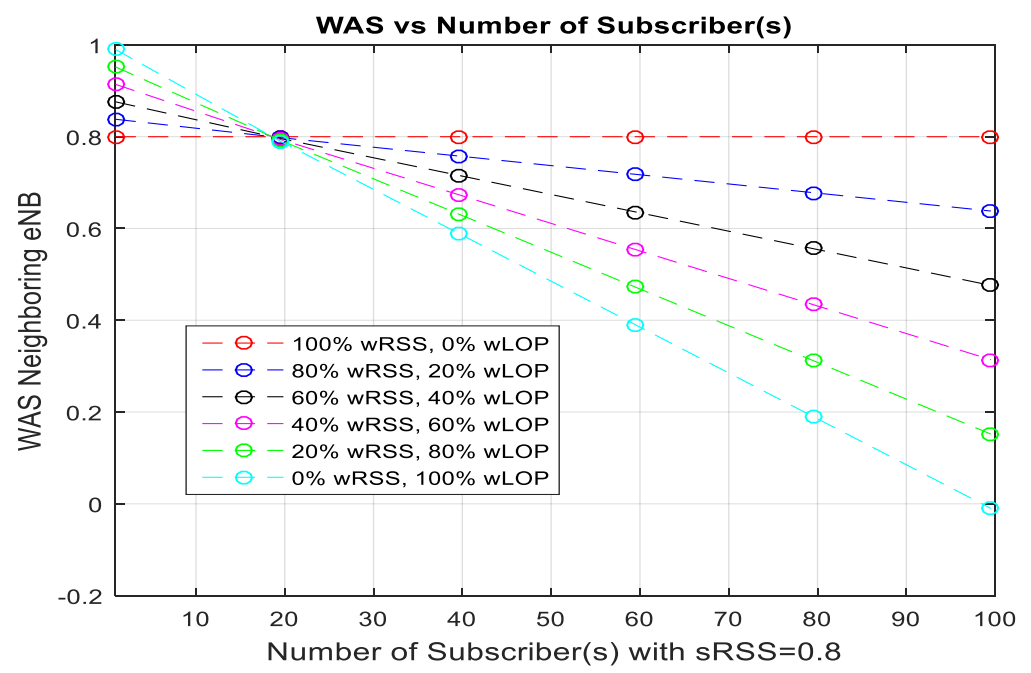

Figure 2. WAS neighboring eNB vs number of subscribers

\section{CONCLUSION}

As a conclusion, this paper introduced two parameters that can improve the user's performance, avoid traffic congestion and prolong battery life of base station for natural disaster condition. The parameters are known as RSS and left over power LoP. By applying these parameters to the modified SWAS, the UE located near to base station $(\mathrm{Srss}=0.8)$ has improve RSS value and QoS at UE as $\alpha$-coefficient has been introduced to signal strength expression. Condition 1 has been chosen for handover condition during natural disasters that select UE with high RSS's value and has battery usage of base station for $20 \%$ only. For future work, we will propose a handover algorithm with improvement of RSS value and Condition 1 for handover conditions. By applying these handover conditions to the handover algorithm, the affected base station will has the UE 
with good QoS, hence prolong battery life as it limit the users to be overloaded base station. Other users which not meet the requirement will be handover to potential target base stations.

\section{ACKNOWLEDGEMENTS}

This paper is part of research work that supported by Bestari Grant file no.: 600-IRMI/DANA 5/3/BESTARI (120/2018) and Faculty of Electrical Engineering, Universiti Teknologi MARA Shah Alam.

\section{REFERENCES}

[1] U. Zakia, E. Karim, M. W. Turza, T. Z. Moumita, and T. A. Khan, "A Navigation system for rescue operation during disaster management using LTE advanced network and WPAN," in 2016 IEEE 7th Annual Information Technology, Electronics and Mobile Communication Conference (IEMCON), 2016.

[2] L. Baumg and M. Max, "Emergency Communication in Challenged Environments via Unmanned Ground and Aerial Vehicles," in 2017 IEEE Global Humanitarian Technology Conference (GHTC), 2017.

[3] S. G. Fernandez et al., "Unmanned and autonomous ground vehicle," International Journal of Electrical and Computer Engineering (IJECE), vol. 9, no. 5, pp. 4466-4472, 2019.

[4] S. K. Debnath et al., "A review on graph search algorithms for optimal energy efficient path planning for an unmanned air vehicle," Indonesian Journal of Electrical Engineering and Computer Science, vol. 15, no. 2, pp. 743-749, 2019.

[5] A. Yuki, U. Satoshi, S. Ryosuke, and R. Adachi, "Event Detection using Mobile Phone Mass GPS Data and Their Reliavility Verufucation by DMSP/OLS Night Light Image," in ISPRS Annals of the Photogrammetry, Remote Sensing and Spatial Information Sciences, 2016, vol. III-2, pp. 12-19, 2016.

[6] M. Raeesi and A. Sadeghi-Niaraki, "GIS Based System for Post-Earthquake Crisis Management using Cellular Network," Int. Arch. Photogramm. Remote Sens. Spat. Inf. Sci., vol. XL-1/W3, pp. 5-8, 2013.

[7] S. Al-marri and M. Ramachandran, "Global Emergency-Response System Using GIS," in Enterprise Information Systems and Implementing IT Infrastructures: Challenges and Issues, pp. 1604-1611, 2010.

[8] M. Casoni, C. A. Grazia, M. Klapez, N. Patriciello, A. Amditis, and E. Sdongos, "Integration of Satellite and LTE for Disaster Recovery," IEEE Commun. Mag., vol. 53, no. 3, pp. 47-53, 2015.

[9] S. Adibi, "A Mobile Health Network Disaster Management System," in 2015 Seventh International Conference on Ubiquitous and Future Networks, pp. 424-428, 2015.

[10] S. Samadi, J. Alzubi, and O. A. Alzubi, "Optimum Range of Angle Tracking Radars: A Theoretical Computing Optimum range of angle tracking radars: a theoretical computing," International Journal of Electrical and Computer Engineering, vol. 9, no. 3, pp. 1765-1772, 2019.

[11] R.K. Jha, A. Singh, A. Tewari, and P. Shrivastava, "Performance Analysis of Disaster Management using WSN Technology," Procedia Comput. Sci., vol. 49, pp. 162-169, 2015.

[12] J. R. Ragini, P. M. R. Anand, and V. Bhaskar, "Big Data Analytics for Disaster Response and Recovery through Sentiment Analysis," Int. J. Inf. Manage., vol. 42, no. May, pp. 13-24, 2018.

[13] D. Hartama, H. Mawengkang, M. Zarlis, and R. W. Sembiring, "Smart City: Utilization of IT resources to encounter natural disaster," J. Phys. Conf. Ser., vol. 890, pp. 1-6, 2017.

[14] D. Singh, "mKRISHI ® Fisheries: A case study on Early Warning System (EWS) for Disaster Communication and Management," in 2016 IEEE International Symposium on Technology and Society (ISTAS), no. October, pp. 106-111, 2016.

[15] Y. Lien and K. Huang, "Cross Network Topology Design for Contingency Cellular Network," in 2014 IEEE Canada International Humanitarian Technology Conference - (IHTC), 2014.

[16] R. Austin, P. Bull, and S. Buffery, "A Raspberry Pi Based Scalable Software Defined Network Infrastructure for Disaster Relief Communication," in 2017 IEEE 5th International Conference on Future Internet of Things and Cloud, pp. 265-271, 2017.

[17] H. Zhang, C. Jiang, R. Q. Hu, and Y. Qian, "Self-Organization in Disaster-Resilient Heterogeneous Small Cell Networks," IEEE Netw., vol. 30, no. 2, pp. 116-121, 2016.

[18] J. Huang, Y. Wu, and Y. Lien, "Bandwidth Allocation of Contingency Cellular Network," in 2013 16th International Symposium on Wireless Personal Multimedia Communications (WPMC), 2013.

[19] J. Huang, Y. Lien, and C. Wang, "Design of Multi-Path Network Topology for Contingency Cellular Network," in 2015 2nd International Conference on Information and Communication Technologies for Disaster Management (ICT-DM), 2015.

[20] S. K. Ray, Roopak Sinha, and S. K. Ray, "A Smartphone-based Post-Disaster Management Mechanism Using WiFi Tethering," in 2015 IEEE 10th Conference on Industrial Electronics and Applications (IClEA), pp. 966-971, 2015.

[21] K. Ali, H. X. Nguyen, V. Quoc-Tuan, S. Purav, and C. Zheng, "Disaster Management Using D2D Communication with Power Transfer and Clustering Techniques," IEEE Access, vol. 6, pp. 14643-14654, 2018.

[22] H. Gao, Y. Shen, and B. Yang, "D2D Communication for Disaster Recovery in Cellular Networks," in 2017 International Conference on Networking and Network Applications, 2017.

[23] S. K. Ray, W. Liu, H. Sirisena, S. K. Ray, and D. Deka, "An Energy Aware Mobile-Controlled Handover Method for Natural Disaster Situations," in 2013 Australasian Telecommunication Networks and Applications Conference (ATNAC), pp. 130-135, 2013. 
[24] S. K. Ray, N. I. Sarkar, D. Deka, and S. K. Ray, "LTE-Advanced Based Handover Mechanism for Natural Disaster Situations," in 2015 International Conference on Information Networking (ICOIN), pp. 165-170, 2015.

[25] T. Nguyen-duc and E. Kamioka, "An SDN Approach for an Energy-Efficient Heterogeneous Communication Network in Disaster Scenarios," Int. J. Wirel. Mob. Networks, vol. 8, no. 6, pp. 1-18, 2016.

[26] Y. Chen and JieYang, "Defending Against Identity-Based Attacks in Wireless Networks," Handb. Secur. Cyber-Physical Crit. Infrastruct., pp. 191-222, 2012.

[27] A. L. Yusof, A. Eiza, M. Azhar, and N. Ya, "Performance analysis of handover parameter for natural disaster in LTE-a network," vol. 16, no. 1, pp. 319-324, 2019.

[28] A. Merwaday and Ismail Guvens, "UAV Assisted Heterogeneous Networks for Public Safety Communications," in 2015 IEEE Wireless Communications and Networking Conference (WCNC), pp. 329-334, 2015.

\section{BIOGRAPHIES OF AUTHORS}

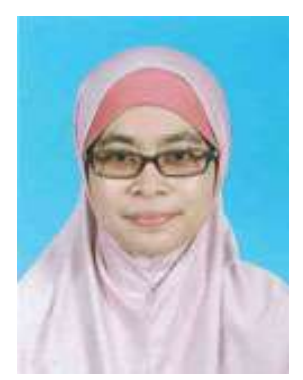

Azita Laily Yusof is an Associate Professor in the Department of Communication Engineering, Universiti Teknologi MARA (UiTM). In 2011, she was awarded a Ph.D degree in Electrical, Electronic \& Systems Engineering from University Kebangsaan Malaysia (UKM). She also obtained her M. Eng. degree from University Kebangsaan Malaysia in Communications \& Computer Engineering in 2000. Previously, she obtained her first degree from University Kebangsaan Malaysia with Honours in Electrical, Electronic \& Systems Engineering in 1999. She is a group member of Wireless Communication Technology Group (WiCoT) at UiTM and a member of IEEE Communications Society. She has been awarded 17 research grants funded by the government and university. She has published over 70 journal papers and conference proceedings on various topics related to wireless communications technologies. Her current research interests include radio resource and interference management in mobile communications networks.

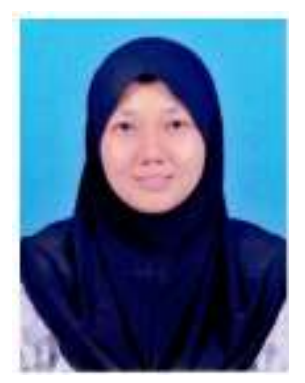

Ainnur Eiza binti Azhar received the Ph.D. degree in Electrical Engineering from the Universiti Teknologi MARA, Malaysia, in 2018. She is currently a Researcher with the Faculty of Electrical Engineering, Universiti Teknologi MARA Shah Alam. Her research interests include PHY channel, interference mitigation technique, LTE-Wi-Fi networks, energy efficiency and green technology. She is a member of Board of Engineer Malaysia and Certified Energy Manager.

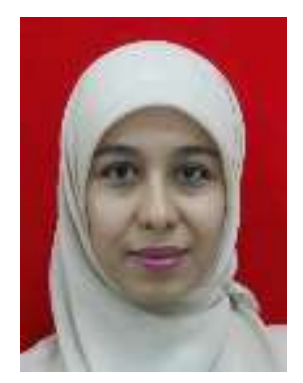

Norsuzila Ya'acob is an Associate Professor in the Department of Communication Engineering, Universiti Teknologi MARA (UiTM). In 2011, she was awarded a Ph.D degree in Electrical, Electronic \& Systems Engineering from University Kebangsaan Malaysia (UKM) for a work on Modeling and Determination of Ionosphere Effects to Improve GPS System Accuracy. She also obtained her M. Sc degree from University Putra Malaysia (UPM) in Remote Sensing and Geographic Information Systems in 2000. Previously, she obtained her B.Eng degree from University Putra Malaysia (UPM), Malaysia in Electronics \& Computer Engineering in 1999. She is a group member of Wireless Communication Technology Group (WiCoT) at UiTM and a member of the IEEE Communications Society. She has published over 120 journal papers and conferences proceeding on various topics related to Satellite, Space Weather, Remote Sensing, and Mobile Communication. Her research interests include Satellite, Space Weather, Remote Sensing, Mobile Communication and Signal Processing. 